BNL-100642-2013-CP

\title{
Generation of Tunable Narrowband Terahertz Pulses from Coherent Transition Radiation
}

\author{
Yuzhen Shen, Xi Yang, G. L. Carr, Richard Heese, Yoshiteru Hidaka, \\ James B. Murphy, and Xijie Wang
}

Presented at the 2012 Conference on Lasers and Electro-Optics (CLEO) (IEEE), San Jose, CA, May 06-11, 2012

Photon Sciences

\section{Brookhaven National Laboratory}

\section{U.S. Department of Energy USDOE - Office of Science}

\footnotetext{
Notice: This manuscript has been authored by employees of Brookhaven Science Associates, LLC under Contract No. DE-AC02-98CH10886 with the U.S. Department of Energy. The publisher by accepting the manuscript for publication acknowledges that the United States Government retains a non-exclusive, paid-up, irrevocable, world-wide license to publish or reproduce the published form of this manuscript, or allow others to do so, for United States Government purposes.

This preprint is intended for publication in a journal or proceedings. Since changes may be made before publication, it may not be cited or reproduced without the author's permission.
} 


\section{DISCLAIMER}

This report was prepared as an account of work sponsored by an agency of the United States Government. Neither the United States Government nor any agency thereof, nor any of their employees, nor any of their contractors, subcontractors, or their employees, makes any warranty, express or implied, or assumes any legal liability or responsibility for the accuracy, completeness, or any third party's use or the results of such use of any information, apparatus, product, or process disclosed, or represents that its use would not infringe privately owned rights. Reference herein to any specific commercial product, process, or service by trade name, trademark, manufacturer, or otherwise, does not necessarily constitute or imply its endorsement, recommendation, or favoring by the United States Government or any agency thereof or its contractors or subcontractors. The views and opinions of authors expressed herein do not necessarily state or reflect those of the United States Government or any agency thereof. 


\title{
Generation of Tunable Narrowband Terahertz Pulses from Coherent Transition Radiation
}

\author{
Yuzhen Shen, Xi Yang, G. L. Carr, Richard Heese, Yoshiteru Hidaka, James B. Murphy, and Xijie Wang \\ National Synchrotron Light Source, Brookhaven National Laboratory, Upton, NY 11973
}

We demonstrate the generation of tunable, narrow-band, few-cycle and multi-cycle terahertz pulses from a temporally modulated relativistic electron beam through coherent transition radiation.

OCIS codes: 320.0320

Tunable THz radiation with a narrow spectral line width is of great interest for molecular spectroscopy, remote sensing, and imaging. A major obstacle to the wide application of accelerator-based $\mathrm{THz}$ sources is the lack of frequency tunability. This comes from the fact that the THz radiation is generated from a single electron bunch of $\sim 1$ ps duration, which results in a single-cycle or near single-cycle $\mathrm{THz}$ pulse. In this work, we demonstrate both theoretically and experimentally, by using a temporally shaped photocathode drive laser pulse, it is possible to control and manipulate the longitudinal charge distribution of a relativistic electron beam, and thereby generate tunable, narrow-band, few-cycle and multi-cycle THz pulses through coherent transition radiation. The result further widens the potential of existing accelerator facilities as sources of $\mathrm{THz}$ radiation.

The THz source presented in this work is developed at Brookhaven National Laboratory. A magnesium photocathode in a RF photoinjector is illuminated by a temporally modulated laser pulse at 265nm using chirped pulse beating technique. The drive laser pulse is given by

$I(t, \tau)=|E(t)+E(t+\tau)|^{2}=I(t)+I(t+\tau)+2 \sqrt{I(t) I(t+\tau)} \operatorname{Cos}\left(\omega_{0} \tau+b \tau^{2}+2 b \tau t\right)$

It is modulated by a cosine function with a center frequency of $\mu=2 b \tau$, which can be easily tuned by varying the time delay $\tau$ or the laser pulse frequency chirp $b$. Fig. 1 shows the cross-correlation measurements of the temporal profiles of the cathode drive laser pulses for two different time delays. The modulation periods are 1.38 and $0.6 \mathrm{ps}$, corresponding to beat frequencies of 0.73 and $1.67 \mathrm{THz}$.

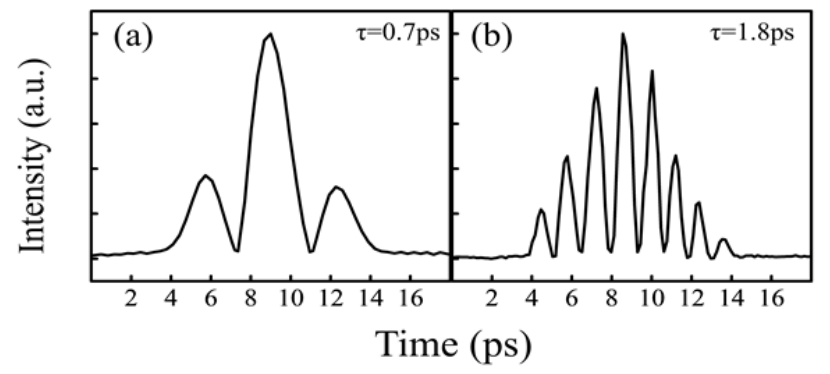

Fig. 1. Temporal profiles of the photocathode drive laser pulses for two different time delays.

The electron bunch is generated from the $5 \mathrm{MeV}$ photocathode, and then accelerated to the relativistic energy of $\sim 120 \mathrm{MeV}$ in two linac sections. Space charge effects play a fundamental role in preservation of the temporal structure of the electron bunch. Fig.2 shows the simulation results of a train of electron bunches which contain 100pC and 400pC, respectively. With $\sim 100 \mathrm{pC}$ charge, the space-charge effect can be minimized by the rapid acceleration of the electron bunch to a relativistic energy, and the temporal structure of the electron bunch can be maintained through the acceleration. As the bunch charge increases further, the density modulation begins to washout because the phase space of each electron bunch grows in both the longitudinal and energy coordinates, leading to the overlap of adjacent bunches. Therefore, $100 \mathrm{pC}$ is used in the experiment, and the zero-phasing technique is applied to measure the longitudinal distribution of electron bunches, which is shown in Fig.3. Projection of this distribution onto the longitudinal coordinate gives the electron density distribution. Due to the bunch compression, the modulation frequency of the electron bunch is $\sim 1.3$ times higher than that of the laser pulse. The modulation frequency of the electron bunch can be as high as $\sim 2.6 \mathrm{THz}$. 

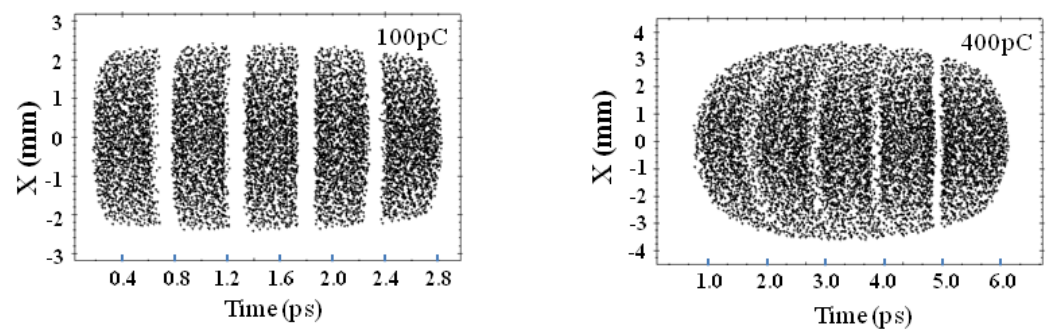

Fig.2. Simulation results of electron distribution in phase space
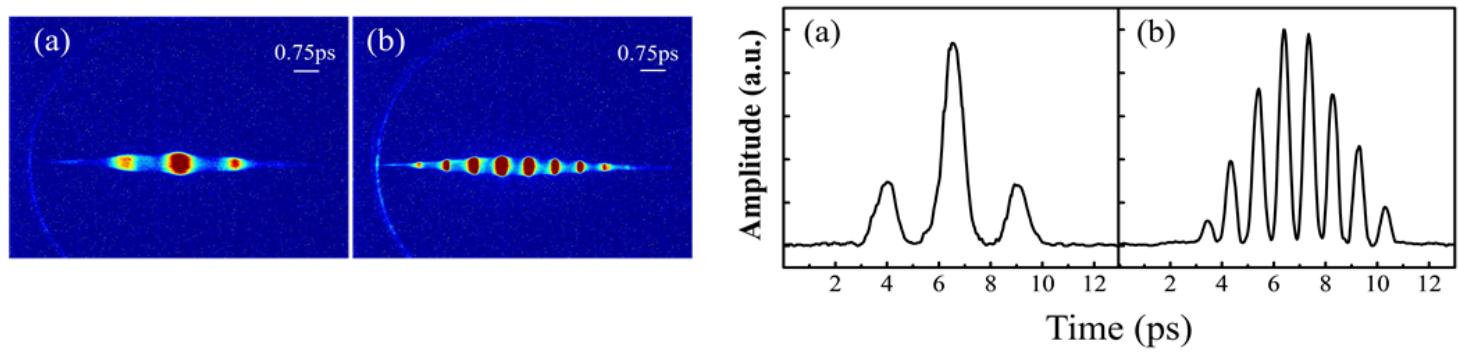

Fig.3. Longitudinal electron bunch distribution (left) and projected electron density distribution (right).

After acceleration, the electron beam is incident onto an aluminum mirror to generate $\mathrm{THz}$ radiation through transition radiation. From equation 1 , the $\mathrm{THz}$ radiation spectrum is given by

$f(\omega)=4 \exp \left(-\alpha \tau^{2}\right) \exp \left(-\frac{(\omega-\mu)^{2}}{4 \alpha}\right)$

which indicates coherent radiation at the modulation frequency $\mu$ for each delay $\tau$, and therefore the tunable coherent $\mathrm{THz}$ radiation can be obtained by using the photocathode drive laser to apply a quasisinusoidal modulation on the electron bunch at $\mathrm{THz}$ frequencies. From equations 2, the radiation energy will drop with increasing the time delay and the THz frequencies.

The $\mathrm{THz}$ radiation is measured with a step-scan Michelson interferometer. Fig. 4 shows the $\mathrm{THz}$ interferograms obtained for different laser and electron bunch profiles shown in Figs. 1 and 3. The Fourier transform of the interferogram gives the power spectra of $\mathrm{THz}$ radiation, as shown in Fig. 5 . The THz radiation is narrow-band with central frequencies at 0.4 and $1.1 \mathrm{THz}$, consistent with the modulation frequency of the electron bunch. The spectral bandwidth for different central wavelengths is $\sim 0.16 \mathrm{THz}$. A train of $\mathrm{N}$ electron bunches whose amplitude is quasi-sinusoidally modulated results in an interferogram with $2 \mathrm{~N}-1$ peaks and gives rise to $\mathrm{N}$-cycles of the electromagnetic field. The central frequency of the $\mathrm{THz}$ spectrum is tunable from $\sim 0.26$ to $2.6 \mathrm{THz}$, which is limited by space-charge effect, and the $\mathrm{THz}$ energy is measured to be $\sim 0.5$ to $2.5 \mathrm{uJ}$ within the frequency tunable range.

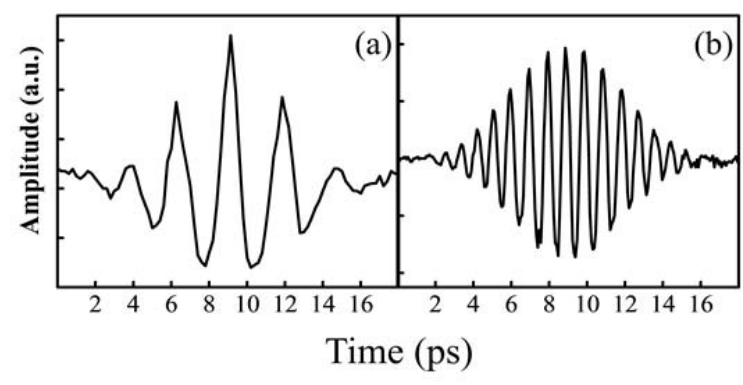

Fig. 4. Field autocorrelation of $\mathrm{THz}$ radiation

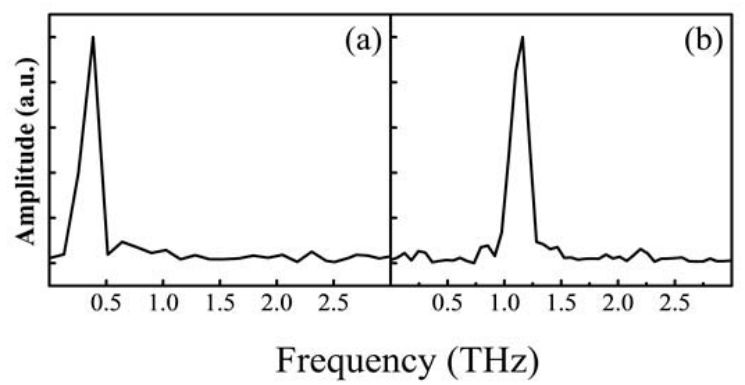

Fig.5. Spectra of THz radiation

In summary, we demonstrate the generation and characterization of tunable narrowband $\mathrm{THz}$ pulses from coherent transition radiation. The central frequency of the THz spectrum is tunable from $\sim 0.26$ to $2.6 \mathrm{THz}$ with a bandwidth of $\sim 0.16 \mathrm{THz}$. 